\title{
Intermittent search strategies revisited: effect of the jump-length and biased motion
}

\author{
F. Rojo, ${ }^{1}$ J. Revelli, ${ }^{2}$ C. E. Budde,${ }^{1}$ H. S. Wio, ${ }^{2}$ G. Oshanin,${ }^{3}$ and K. Lindenberg ${ }^{4}$ \\ ${ }^{1}$ Fa.M.A.F., Universidad Nacional de Córdoba, \\ Ciudad Universitaria, X5000HUA Córdoba, Argentina \\ ${ }^{2}$ Instituto de Física de Cantabria, Universidad de Cantabria and CSIC \\ E-39005 Santander, Spain \\ ${ }^{3}$ Laboratoire de Physique Thèorique de la Matière Condensée, \\ Université Pierre et Marie Curie, 4 place Jussieu, 75252 Paris Cedex 5 France \\ ${ }^{4}$ Department of Chemistry and Biochemistry, \\ University of California, San Diego, La Jolla, CA 92093-0340 USA
}

\begin{abstract}
We study the kinetics of search of a single fixed target by a large number of searchers performing an intermittent biased random-walk in a homogeneous medium. The states of motion are essentially characterized by the length of the jump and the probability to make a step in a given direction. By considering such a framework, we propose a model describing the searchers' dynamics, generalizing results of previous work. We have obtained, and numerically evaluated, theoretical results for the mean number of total distinct visited sites up to a maximum evolution time, and have also studied the dependence of this quantity on both, the transition probability between the states and the length of the jump on each state. In addition to the theoretical approach, we have implemented Monte Carlo simulations, finding an excellent agreement between the theoretical-numerical and simulations results.
\end{abstract}

PACS numbers: 05.45.-a, 05.40.Ca, 82.40.Ck 


\section{INTRODUCTION}

The question of optimal search strategies has motivated a great deal of work during the last few years [1-3]. Among other aspects, the determination of an efficient search strategy emerged as a crucial problem in behavioral ecology, inspiring numerous experimental and

theoretical works [4-6]. It is also relevant to broader domains such as stochastic processes theory, applied mathematics and molecular biology as well as in social sciences and ecology $[7-17]$.

In addition to deterministic trajectories, random trajectories have proved to play a crucial role in many search processes (involving for instance a searcher with fewer abilities). In particular, it has been shown that among the class of randomly reoriented ballistic trajectories, Levy flights can optimize the encounter rate with random targets [13, 14]. However, Levy strategies are optimal only in the particular case of non destructive search, while in the case of destructive search, where each target definitely disappears after the first encounter, or in the case of single target, it is possible to show [10] that the trajectories optimizing the encounter rate with targets are simply linear ballistic motions, and therefore not of Levy type. In opposition to such Levy strategies, it has been observed that numerous animal species $[7,10,11]$ switch between two distinct type of behavior (and motion) while foraging. In fact, many foragers like plankitovorous fish [18], ground foraging birds and lizards [4-6], adopt an intermittent searching behavior. These intermittent search strategies combine phases of relocation (in which the searcher may or may not capture the target), and a thorough search phase, which allow for target detection. The modeling of such an intermittent search strategy was studied in the context of the trapping problem in [7, 15-17], where the observable optimized was the mean first-passage time. When dealing with the target problem the survival probability, that is the probability that the target [11] remains undetected up to the maximal search time $t$; can be optimized as a function of the transition rates among the internal states representing different types of motion.

In previous work [9-11] we have presented a theoretical model for the intermittent search kinetics of a hidden target. Here we revisit the so called target problem, in which we have a single static target and a set of diffusing searchers, initially distributed in the field and go one step forward considering several types of foraging motion. Firstly, we consider the effect of the jump-length on the proposed search process and secondly we study the influence of 
anisotropy in the displacement. We set up our scheme exploiting the multi-state randomwalk (RW) formalism developed by Montroll and Weiss [19, 20].

The outline of this paper is as follows. In the next section we present a few results from the multi-state Random Walk and definitions of the functions to be used latter. In Section III we describe our model, focusing on the one dimensional analytical solvable case and made the connection with Monte Carlo simulations. In Section IV we present some results for the survival probability and related functions, while in the last Section we draw some conclusions.

\section{SOME RESULTS FROM MULTI-STATE RANDOM WALK TECHNIQUE}

Here we recall a few basic results from the formalism developed by Weiss and Montroll $[11,19,20]$ that are useful for our objectives. We assume that at time $t$ each walker can be at site $s$ on a 1D lattice, in one of two internal states and that the transition between the internal states are exponential functions of time with parameters $\gamma_{1}$ and $\gamma_{2}$. In addition, as initial conditions, we adopt that at $t=0$ the walker is at $s=0$, with a probability $g_{i}$ $(i=1,2)$ of being in state $i$. Taking these assumptions into account we can write the joint conditional probability of being at site $s$ in the internal state $j(j=1,2)$, as

$$
\begin{aligned}
& P_{1}(s, t \mid 0, t=0)=g_{1} P_{11}(s, t \mid 0, t=0)+g_{2} P_{12}(s, t \mid 0, t=0), \\
& P_{2}(s, t \mid 0, t=0)=g_{1} P_{21}(s, t \mid 0, t=0)+g_{2} P_{22}(s, t \mid 0, t=0),
\end{aligned}
$$

where $P_{i j}(s, t \mid 0, t=0)$ is the joint conditional probability of being at site $s$, in the internal state $i$ at time $t$, given that at $t=0$ it was at $s=0$ in internal state $j$. The exploitation of the indicated formalism leads us to analytic closed expressions for $P_{i j}(s, t \mid 0, t=0)$ in the Fourier-Laplace space (we will keep track of transformations by explicitly writing the arguments of all functions). The relevant transformed results reads

$$
\begin{aligned}
& \hat{P}_{11}(k, u \mid 0, t=0)=\frac{u+\gamma_{2}-\lambda_{2}\left(p_{2}(k)-1\right)}{D(k, u)} \\
& \hat{P}_{12}(k, u \mid 0, t=0)=\frac{\gamma_{2}}{D(k, u)} \\
& \hat{P}_{21}(k, u \mid 0, t=0)=\frac{\gamma_{1}}{D(k, u)} \\
& \hat{P}_{22}(k, u \mid 0, t=0)=\frac{u+\gamma_{1}-\lambda_{1}\left(p_{1}(k)-1\right)}{D(k, u)}
\end{aligned}
$$


where

$$
D(k, u)=\left(u+\gamma_{2}-\lambda_{2}\left(p_{2}(k)-1\right)\right)\left(u+\gamma_{1}-\lambda_{1}\left(p_{1}(k)-1\right)\right)-\gamma_{1} \gamma_{2}
$$

and $p_{j}(k)$ is the lattice's structure factor for state $j, \lambda_{i}$ being the diffusion parameter in state $i$.

\section{THE MODEL}

As it was done in [9-11] we restrict ourselves to work on an infinite discrete chain, and we also assume that the fixed target is located at the origin of the lattice. We assume that the searcher has "a general tendency" (i.e. a preferred direction of motion, not necessarily pointing towards the prey) that depends on its internal state. We include such a foraging behavior into our model as a biased walk.

In the following we assume that the searcher alternatively displays two distinct search behaviors

- a search phase, hereafter referred to as phase 1, during which the searcher makes a compact exploration of its local area. This local scanning is modeled as a slow diffusive motion. Eventually the target is found when this movement reaches the target location for the first time.

- a relocation phase, referred to as phase 2, during which the searcher explores more distant regions of the lattice. However, the target is found if the predator lands over it.

At $t=0$, we assume that a uniform distribution of searchers/predators starts the "search". The first one that finds the target/prey, catches it with probability one (i.e. perfect trapping). Each predator makes two type of motion on the lattice (see Fig. 1)

- state-1 (search phase), corresponding to a RW with jumps to the 1st neighbors, with a rate $\lambda_{1}=\lambda$

- state-2 (relocation phase), also corresponding to a RW with jumps to the $N$-th neighbors, with a rate $\lambda_{2}=\lambda$. 


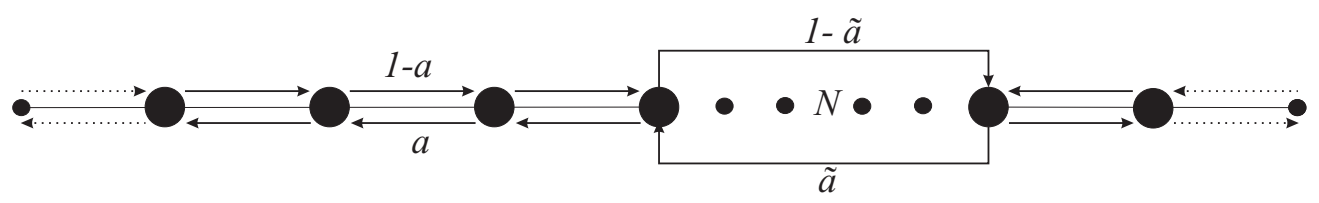

FIG. 1: System's draft

Let us assume that we have a separable RW. In addition, in one dimension $p_{1}(k)=$ $\cos (k)+i(1-2 a) \sin (k)$ and $p_{2}(k)=\cos (N k)+i(1-2 \tilde{a}) \sin (N k)$. The coefficients $a, \tilde{a}$ determines the jumping asymmetry along the lattice in each state.

In a homogeneous system, the survival probability $\phi(t)[9,19-21]$ may be written as $\phi(t)=e^{-\rho S(t)}$, where $S(t)$ corresponds to the mean number of distinct sites visited up to time $t$, and $\rho$ is the initial occupation probability of each site (that is, the predators' density). It was shown [21] that when the target detection occurs in any of the two internal states

$$
S_{M a r}(u)=\frac{1}{u^{2} P_{M a r}(s=0, u \mid 0, t=0)},
$$

and $P_{\text {Mar }}(s, t \mid 0, t=0)$ must be interpreted as the marginal probability of finding the predator at site $s$ at time $t$, independently of the internal state

$$
P_{M a r}(s, t \mid 0, t=0)=P_{1}(s, t \mid 0, t=0)+P_{2}(s, t \mid 0, t=0) .
$$

In the high transition rate regime between internal states [11](with $\left(\gamma_{1}+\gamma_{2}\right) \gg \lambda$ and $\left.u \ll\left(\gamma_{1}+\gamma_{2}\right)\right) P_{M a r}(s, u \mid 0, t=0)$ may be expressed as

$$
P_{M a r}(s, u \mid 0, t=0)=\frac{1}{2 \pi} \int_{-\pi}^{\pi} \frac{e^{i k s} d k}{(1+u)-\left(\frac{\gamma_{2}}{\gamma_{1}+\gamma_{2}}\right) p_{1}(k)-\left(1-\frac{\gamma_{2}}{\gamma_{1}+\gamma_{2}}\right) p_{2}(N k)}
$$

where the $\gamma_{i}$ are measured in units of $\lambda$. Denoting $\alpha=\frac{\gamma_{1}}{\gamma_{1}+\gamma_{2}}, z=\frac{1}{u+1}, b=1-2 a$ and $\tilde{b}=1-2 \tilde{a}$ we can write

$$
P_{M a r}(s, u \mid 0, t=0)=\frac{z}{2 \pi} \int_{-\pi}^{\pi} \frac{e^{i k s} d k}{1-z[(1-\alpha)(\cos (k)+i b \sin (k))+\alpha(\cos (N k)+i \tilde{b} \sin (N k))]}
$$

This expression corresponds to a slight generalization of Eq. (4.4) in Ref.[9] or Eq. (15) in [11]. 


\section{A. The Effect of the Jump-Length in the search process}

Here we consider the effect of the jump-length in the proposed search process, leaving the displacement's anisotropy for the next paragraph. In this case we assume $b=\tilde{b}=0$, hence

$$
P_{M a r}(s, u \mid 0, t=0)=\frac{z}{\pi} \int_{0}^{\pi} \frac{\cos (k s) d k}{1-z[(1-\alpha) \cos (k)+\alpha \cos (N k)]}
$$

Calling $k_{i}$ to the roots of

$$
1-z[(1-\alpha) \cos (k)+\alpha \cos (N k)]=0
$$

and using the identity

$$
2 \cos (N k)=(2 \cos (k))^{N}-N(2 \cos (k))^{N-2}+\frac{N}{2}\left(\begin{array}{c}
N-3 \\
1
\end{array}\right)(2 \cos (k))^{N-4}-\ldots,
$$

Eq. (6) could be written as

$$
P_{M a r}(s, u \mid 0, t=0)=\frac{1}{2^{N-1} \alpha} \sum_{i=1}^{N} A_{i} \int_{0}^{\pi} \frac{\cos (k s)}{\left(\cos (k)-\cos \left(k_{i}\right)\right)} d k,
$$

where the coefficients $A_{i}$ are expressed in terms of the $k_{i}$.

The evaluation of the integral up to $N=4$, although tedious, is straightforward and can be done analytically. The results, after performing the Laplace inversion (numerically), are shown in Section IV. Results for $N>4$ are also shown but in this case only trough Monte Carlo Simulations.

\section{B. "Drift Controlled" Random Walks}

We now consider a certain degree of anisotropy in the displacement of the Random Walk. In order to simplify the analysis, hereafter we will only consider jumps to next nearest neighbors $(N=2)$ in state 2 .

The general case with arbitrary values of $b$ and $\tilde{b}$ is treated in the Appendix. Besides this, if we have the same anisotropy in both states $(b=\tilde{b})$, and as long as $b \ll 1\left(a \simeq \frac{1}{2}\right)$ we can make a perturbation analysis of Eq. (5) in terms of $b$ as

$$
\begin{aligned}
P_{M a r}(s, u \mid 0, t=0)=\frac{1}{\pi} & \left.\int_{0}^{\pi} \cos (k s) P_{M a r}(k, u \mid 0, t=0)\right|_{b=0} d k \\
& +\left.\frac{b^{2}}{\pi} \int_{0}^{\pi} \cos (k s) \frac{\partial^{2} P_{M a r}(k, u \mid 0, t=0)}{\partial b^{2}}\right|_{b=0} d k+\ldots
\end{aligned}
$$


Due to the symmetry, and once valuated in $b=0$, odd terms in $b$ disappear from the integrand. The results, after performing the numerical Laplace inversion, are shown in the next section.

\section{RESULTS}

In order to test our theoretical results we have made a comparison with Monte Carlo simulations. To evaluate the theoretical expressions we have used a numerical procedure to calculate the inverse Laplace transform [22]. Since there are too many parameters to set up the system, we have fixed some of them. For the initial condition we have chosen $g_{1}=g_{2}=1 / 2$ and the evolution time was set equal to $t=100$. Regarding the Monte Carlo simulations, the process is such that at each click of the clock the searcher took the decision to jump into state 1 or 2 with transition probability $\alpha$ or $1-\alpha$. We have averaged over $10^{5}$ realizations.

\section{Jump-Length in the search process}

In Fig. 2 we show $S(t)$, the mean number of distinct visited sites, for different jump lengths on the second motion state as a function of $\alpha$. From the figure we can see that there is an excellent agreement between the theoretical-numerical results and Monte Carlo simulations. From this figure it is also apparent that the maximum mean number of visited sites varies according to the jump length. This behavior is well described in Fig.3 where we have depicted the maximum mean number of visited sites as a function of the jump length on the state of motion 2. This figure shows a curve increasing towards a stationary value which could be understood considering that when the jump length increases then the possibility to make an overlap to the already visited sites (in the other state) is low.

\section{"Drift Controlled" Random Walk}

The case with small bias, that is a small departure from the case analyzed in [11] is shown in Fig. 4. There we depict both numerical analytical results and Monte Carlo simulations, finding (once again) an excellent agreement between the two results. The figure shows that 


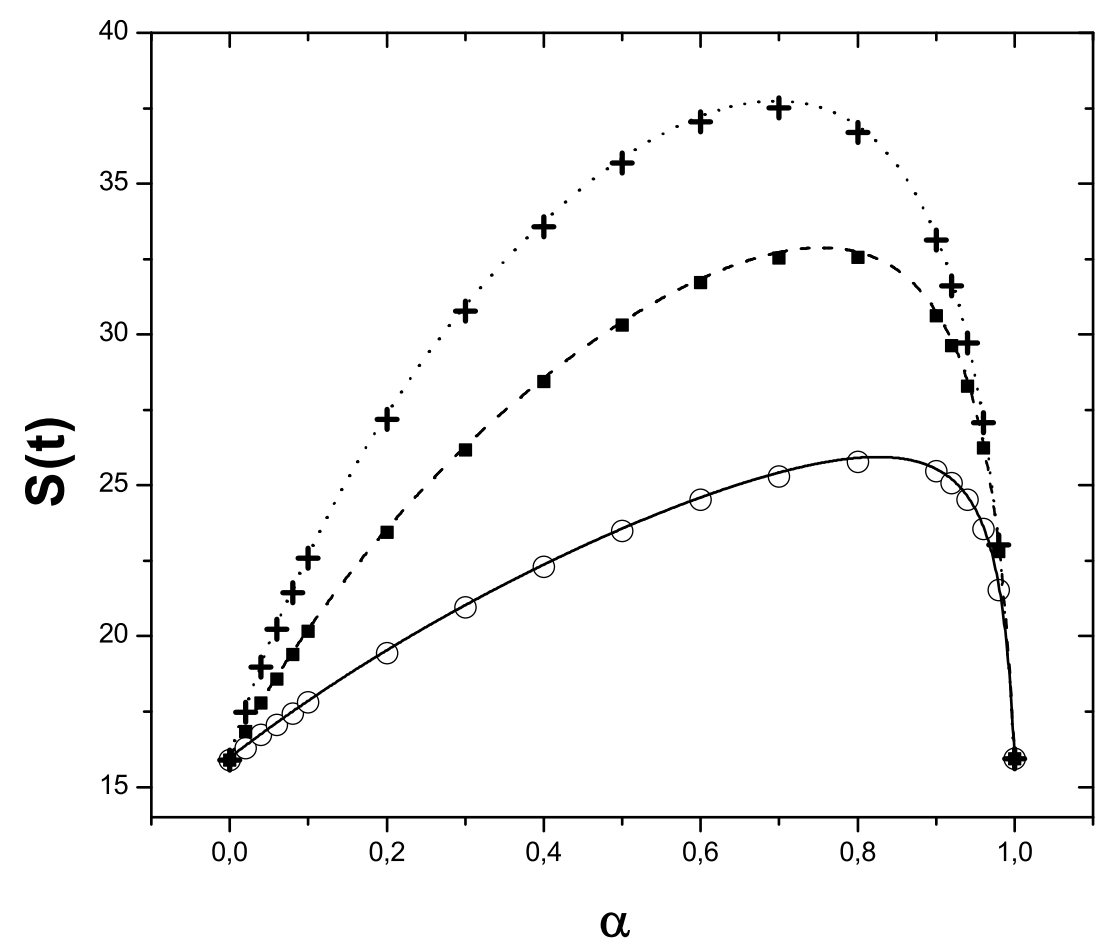

FIG. 2: Total mean number of distinct visited sites up to time $t=100$. Lines represent numerical calculations, symbols represent Monte Carlo simulations. Circles represents the case of jumps length (in the 2nd state of motion) to second neighbors, squares to third neighbors and crosses to fourth neighbors.

small deviations from the situation without bias produce small departures in the response, according to the predictions of theory.

In figure 5-a we draw the phase diagram that summarize the behavior of the system in the bias case for a fixed time $t=100$. The diagram is plotted as a function of $\alpha$ (the parameter that regulates the intermittency) and the bias difference $(a-\tilde{a})$. In grey scale is shown the mean number of distinct visited sites; darker means a larger value in $S(\alpha, a-\tilde{a} ; t=100)$. In this figure we only show positive values of $(a-\tilde{a}),-(a-\tilde{a})$ generates the same diagram.

In order to study the effect of bias, in Fig 5-b and 5-c we show cuts of $S(\alpha, a-\tilde{a} ; t=100)$. Each section (I-VI) correspond to a curve in Fig. 5-b and 5-c. Here we depict the case where 

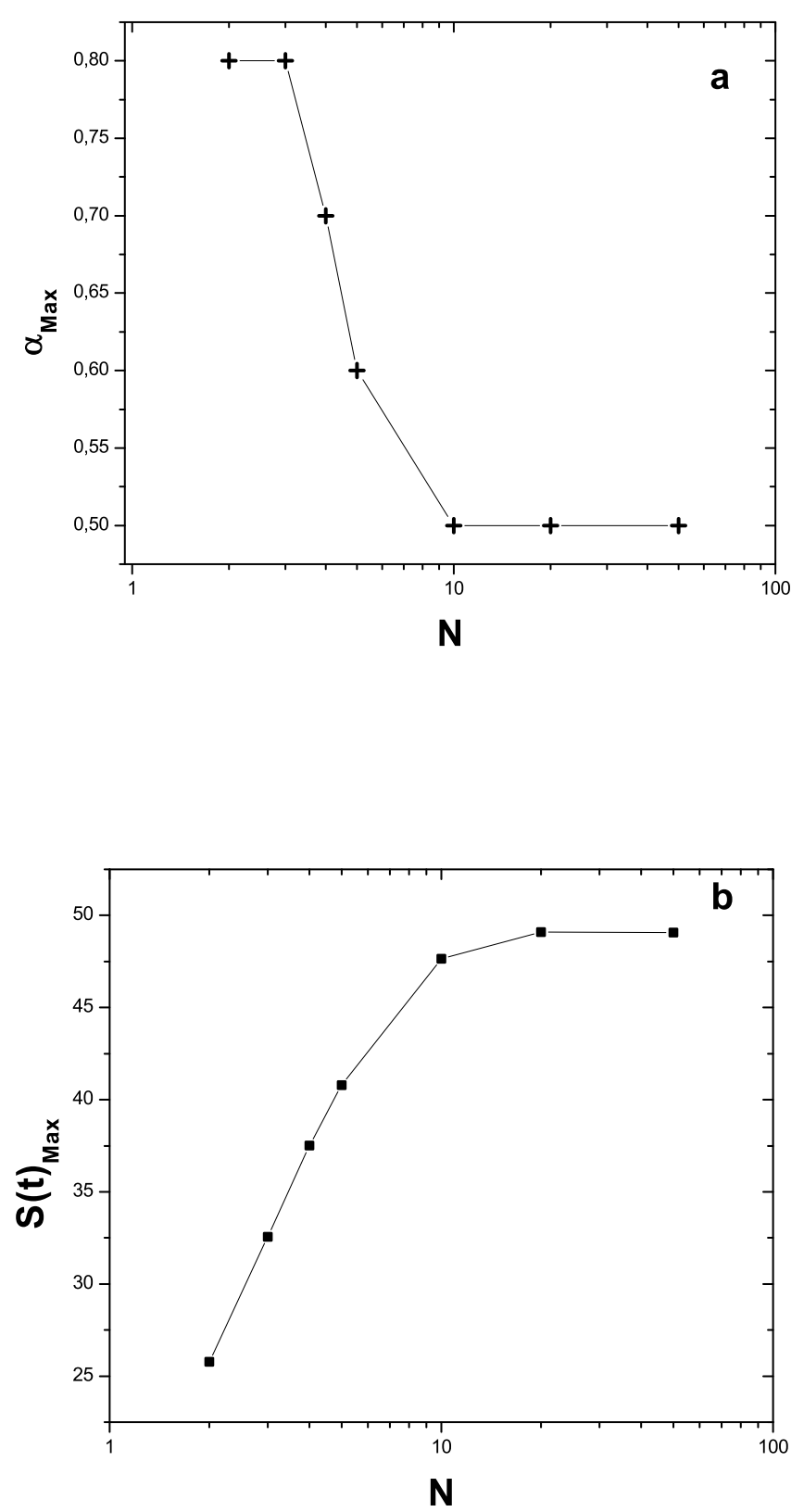

FIG. 3: Transition probability from state 1 to state 2, and total mean number of distinct visited sites that occur at the maximum $\alpha$ value, as function of the jump's length $N$. All data corresponds to Monte Carlo simulations.

there is a strong bias on state of motion 1 and we compare among different forms of motion on state 2. These curves show a richer dynamics. When the bias on the state of motion 2 


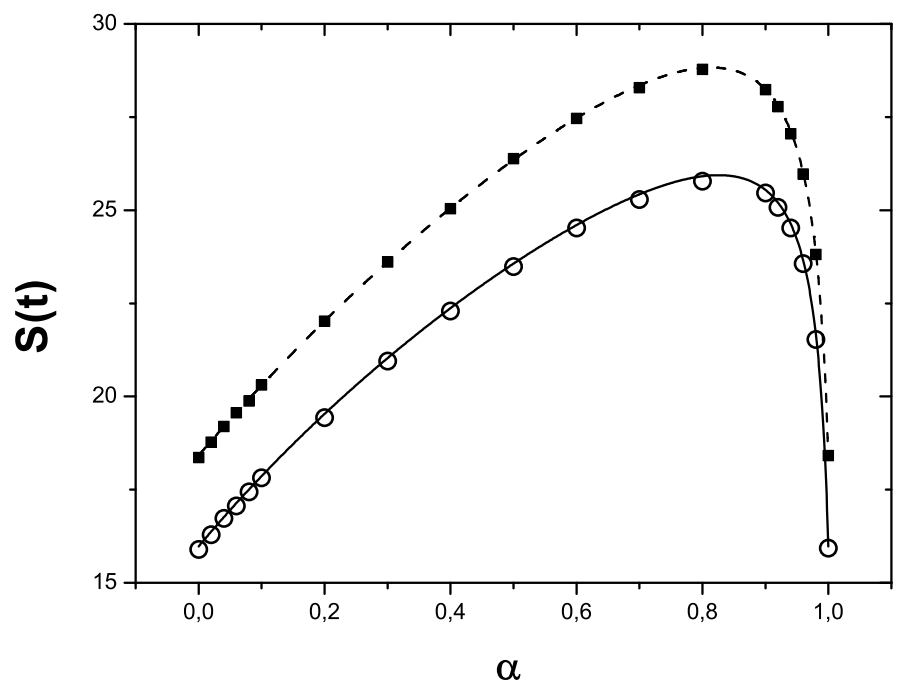

FIG. 4: Total mean number of distinct visited sites for small bias at time $t=100$. Lines represent numerical calculations, symbols represent Monte Carlo simulations: circles correspond to no bias case, while black squares are for $a=0.55$.

is opposite to the one in the state of motion 1 then the curves show a local maximum and a local minimum (curves I and II in Fig 5-b). It is a novel result because up to now the responses obtained on this kind of systems had shown only a non monotonous behavior, but with only a maximum. When bias on the state of motion 2 is decreased (see for example the case $\tilde{a}=0.45)$ then the maximum disappear and a small plateau of constant $S(t)$ takes place (curve III). When the bias on the state motion 2 increases in the same direction to the state of motion 1 the curves decreases in a monotonous way (curves IV and V in Fig. 5-c). However, when the values of both bias are the same or almost the same, the predicted maximum situation is reobtained again (curve VI). It is worth remarking here the excellent agreement between the analytical-numerical results and the Monte Carlo simulations.

The minimum obtained in Fig. 6-a could be understood in the following way,

- the walker in both states has a general tendency (bias) that point in opposite directions, i.e., the tendency in one state is against the one in the remaining state.

- the cases $\alpha \sim 0, \alpha \sim 1$ (single state movement), are straightforward to analyze. These correspond to the searcher being almost all of its time in state 1 or 2 respectively and 

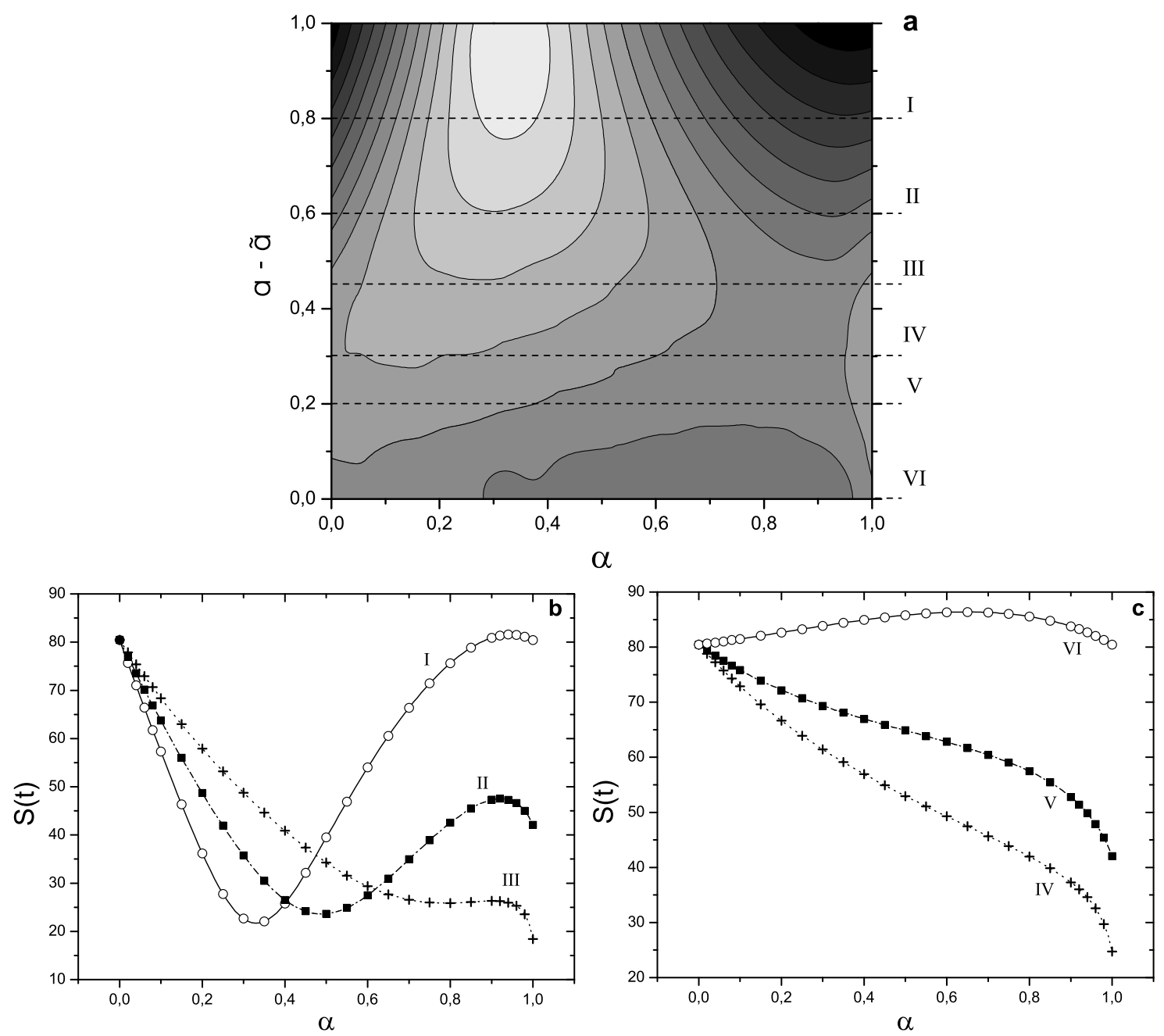

FIG. 5: (a) Phase diagram of the system in the bias case for a fixed time $t=100$ as a function of $\alpha$ (the parameter that regulates the intermittency) and the bias difference $(a-\tilde{a})$. In this figure darker means a larger value in $S(\alpha, a-\tilde{a} ; t=100)$ Numerical calculations (lines) and Monte Carlo simulations (symbols) for the total mean number of distinct visited sites, up to time $t=100$, for a quasi ballistic movement in state $1(a=0.9)$. (b) Biased movement in state 2 to the right, circles $\tilde{a}=0.1$ (I), squares $\tilde{a}=0.3$ (II), crosses $\tilde{a}=0.45$ (III). (c) Biased movement to the left, crosses $\tilde{a}=0.6(\mathrm{IV})$, squares $\tilde{a}=0.7(\mathrm{~V})$, circles $\tilde{a}=0.9(\mathrm{VI})$.

since both are biased, essentially $([19]) S_{\alpha \sim 0}(t) \sim|1-2 a| t$ and $S_{\alpha \sim 1}(t) \sim|1-2 \tilde{a}| t$ as Fig 5-b shows.

- for the minimum behavior $(\alpha<0.5)$ the walker remains most, but not all, of its time in the search phase. In this state (jumps to first neighbors) a compact exploration is 
made; every site is visited so each transition to state 2 is likely to end in an already visited site (by state 1), contributing in this way, to a minimum in the mean number of distinct visited sites.

One might ask why a minimum is not reached for values of $\alpha>0.5$. This could be answered considering the way in which the space is covered by the searcher. For these $\alpha$ values the walker remains most of its time in the re-localization phase (jumps to next nearest neighbors). In this case the pattern of visited sites left by the searcher may be thought as a "strainer". This could be interpreted as a defective way of covering territory, however as transitions to state 1 (jump to nearest neighbors) are allowed, these excursions are likely to fill the gaps left by the walker on its journey on state 2 . Thus transitions in this regime $(\alpha>0.5)$ contribute positively increasing the mean number of distinct visited sites.

\section{CONCLUSIONS}

We have presented a simple model for the kinetics of search, by a set of searchers performing intermittent motion, of a target hidden at the origin of a chain. It is based only on diffusion and complements previous related results given in [9-11]. Here we have considered both: the influence of anisotropy in the displacement of the searcher and studied the effect of the jump-length on the proposed search process.

The intermittency has been characterized by the probability density functions for jumps between the internal states. For simplicity, we have restricted the analysis of intermittency to time exponential functions with parameters $\gamma_{1}$ and $\gamma_{2}$. However, the present framework is completely general.

The present generalization of the work in [11] allowed us to obtain a very rich dynamics for the system's behavior, passing from a single minimum, through a monotonous behavior, to situations with both a local maximum and a local minimum, and finally to a single maximum. All these results were predicted by the "theory" and supported by Monte Carlo simulations, with an excellent agreement between the numerical and simulations results.

Clearly, the present model of intermittent search can be generalized in several directions: higher dimensions, finite size domain systems, continuous systems, imperfect detection, dy- 
namical behavior of the target, non-Markovian transitions between internal states, etc. All these aspects will be the subject of further work.

\section{Acknowledgments}

CB and FR acknowledge financial support by CONICET and SECYT-UNC, Argentina. JR and HSW acknowledge financial support from MEC, Spain, through CGL200764387/CLI. The international collaboration has been facilitated by AECID, Spain, through Projects A/013666/07 and A/018685/08. The research of GO is partially supported by Agence Nationale de la Recherche (ANR) under grant DYOPRI-Dynamique et Optimisation des Processus de Transport Intermittents.

\section{APPENDIX: "DRIFT CONTROLLED" RANDOM WALK}

Here we describe some essential details of section (III B). In order to simplify the analysis we will consider jumps to next nearest neighbors $(N=2)$ in state 2.We start with Eq. (5), and adopt the notation $P_{M a r}(s, u \mid 0, t=0) \equiv P_{M a r}(s, u)$ :

$$
P_{\text {Mar }}(s, u)=\frac{z}{2 \pi} \int_{-\pi}^{\pi} \frac{e^{i k s} d k}{1-z[\alpha(\cos (k)+i b \sin (k))+(1-\alpha)(\cos (2 k)+i \tilde{b} \sin (2 k))]}
$$

Defining $\nu=\exp (i k)$ and using the known relations $\cos (N k)=\frac{\exp (i N k)+\exp (-i N k)}{2}$, $\sin (N k)=\frac{\exp (i N k)-\exp (-i N k)}{2 i}$, eq.(A.1) could be transformed into an integral along the unit circle in the complex plane

$$
P_{M a r}(s, u)=\frac{1}{2 \pi i} \oint_{|\nu|=1} \frac{\nu^{s+1} d \nu}{\frac{-\alpha(1+\tilde{b})}{2}\left[\nu^{4}+\frac{(1-\alpha)(1+b)}{\alpha(1+\tilde{b})} \nu^{3}-\frac{2}{z \alpha(1+\tilde{b})} \nu^{2}+\frac{(1-\alpha)(1-b)}{\alpha(1+\tilde{b})} \nu+\frac{(1-b)}{(1+\tilde{b})}\right]}
$$

The roots of the denominator in (A.2) could be found using standard procedures [23]. Calling $\nu_{l}$ to the $l$-root we write the last integral as

$$
P_{M a r}(s, u)=-\frac{2}{\alpha(1+\tilde{b})} \frac{1}{2 \pi i} \oint_{|\nu|=1} \prod_{l} \frac{\nu^{s+1}}{\nu-\nu_{l}} d \nu=-\frac{2}{\alpha(1+\tilde{b})} \sum_{l} \frac{A_{l}}{2 \pi i} \oint_{|\nu|=1} \frac{\nu^{s+1}}{\nu-\nu_{l}} d \nu
$$

the last equality holds from expanding the product in partial fractions. The coefficients $A_{l}$ are expressed in terms of the $\nu_{l}$, e.g., $A_{1}=\left[\left(\nu_{1}-\nu_{2}\right)\left(\nu_{1}-\nu_{3}\right)\left(\nu_{1}-\nu_{4}\right)\right]^{-1}, A_{2}=$ 
$\left[\left(\nu_{2}-\nu_{1}\right)\left(\nu_{2}-\nu_{3}\right)\left(\nu_{2}-\nu_{4}\right)\right]^{-1}$ and so on. Considering those roots that lie inside the unitary circle, Eq.(A.3) is ready to be solved by using the Cauchy's Residue Theorem.

Despite being able to obtain analytical results in Laplace space, its size and complexity made its analysis a difficult task. The inversion of the Laplace transform of the results seems to be beyond our possibilities so we have used a numerical procedure to calculate it [22].

\section{REFERENCES}

[1] Stephens DW and Krebs JR 1986 Foraging Theory (Princeton, NJ, Princeton University Press)

[2] Stone LD 1989 Theory of Optimal Search (Arlington, VA, Operations Research of America)

[3] Bell JW 1991 Searching Behavior, The Behavioural Ecology of Finding Resources (Chapman and Hall Animal Behaviour Series, London, Chapman and Hall)

[4] OBrien W J, Browman H I, and Evans B I, Am. Sci.78, 152 (1990).

[5] Kramer D, Mclaughlin R, Amer. Zool., 41, 137 (2001).

[6] McAdam A and Kramer D, Anim. Behav., 55, 109 (1998).

[7] Benichou O et al, Phys. Rev. Lett.94, 198101 (2005).

[8] Benichou O et al, J. Phys.: Condens. Matter 19, 065141 (2007).

[9] Oshanin G, Wio H S, Lindenberg K and Burlatsky S F, J.Phys. Cond. Mat. 19, 065142 (2007).

[10] Oshanin G, Lindenberg K, Wio H S and Burlatsky S F, J.Phys. A 42, (2009).

[11] Rojo F, Budde C E, Wio H S, J. Phys. A: Math. Theor. 42, 125002 (2009)

[12] Reynolds A, Europhys. Lett., 75 (4), pp. 517520 (2006)

[13] Bartumeus F et al, Ecology, 86 (11), pp. 3078-3087 (2005)

[14] Raposo E et al. , Phys. Rev. Lett. 91, 240601 (2003)

[15] Benichou O et al, Europhys. Lett. 75 (2), pp. 349-354 (2006)

[16] James A, Plank M J and Brown R, Phys. Rev. E 78, 051128 (2008)

[17] Lomholt M A, Koren T, Metzler R and Klafter J, PNAS 105,no. 32 11055-11059 (2008)

[18] Reynolds A M, Europhys. Lett. 75 517-520 (2006)

[19] Weiss G H, Aspects and Applications of the Random Walk, in Random Materials and Processes, H E Stanley and E Guyon, Eds. (North Holland, 1994) .

[20] Montroll E and West B: On an Enriched Collection of Stochastic Processes, in Fluctuation 
Phenomena, Montroll E, Lebowitz J, eds. North-Holland (1979).

[21] Haus J and Kehr K, Phys. Rep, 150, 195 (1987).

[22] Kryzhniy V V, "On regularization of numerical inversion of Laplace transforms" , J. Inv. Ill-Posed probl. , 12, No.3,279 (2004)

[23] Handbook of Mathematical Functions, edited by M Abramowitz and I Stegun (Dover, New York, 1965). 